\title{
Percepção dos Acadêmicos de Farmácia Sobre a Atuação do Farmacêutico nas Práticas Integrativas e Complementares em Saúde
}

\author{
Taisa Soares da Silva, ${ }^{1}$ Cristina de Santana Farias, ${ }^{2}$ Felisberto Farias dos Santos, ${ }^{2}$ \\ Irineu Ferreira da Silva Neto, ${ }^{2}$ Ana Emília Formiga Marques ${ }^{3}$
}

\section{RESUMO}

As Práticas Integrativas e Complementares em Saúde (Pics) visam a prestar um cuidado de forma holística, com o intuito de promover a saúde e proporcionar um bom equilíbrio entre tecnologia, ciência e a humanização. Assim, o presente artigo tem como objetivo avaliar a percepção dos futuros egressos do curso de Farmácia sobre o papel do farmacêutico nas Pics. Foi realizada uma pesquisa de campo em uma Faculdade de Farmácia no Cariri Cearense. Atualmente a faculdade é constituída por 10 turmas do curso de Farmácia. A amostra utilizada no estudo foi composta por alunos do 9 e 10 semestre do curso de Farmácia. Os resultados do estudo mostram que $85,3 \%$ dos acadêmicos possuem conhecimento a respeito das Pics, no entanto muitos não se sentem seguros quanto aos seus conhecimentos sobre as Práticas. Foi possível observar que os alunos indicariam a homeopatia, acupuntura, terapia floral e plantas medicinais para tratar determinadas enfermidades, estas podendo ser realizadas por farmacêuticos conforme resoluções específicas. Por fim, considera-se que os resultados deste trabalho possam contribuir agregando valores sobre os achados evidenciados, auxiliando na discussão de novos estudos sobre as Práticas Integrativas e Complementares.

Palavras-chave: Conhecimento; estudantes de farmácia; terapias complementares.

\section{PERCEPTION OF PHARMACY ACADEMICS ON PHARMACEUTICAL PERFORMANCE IN INTEGRATIVE AND COMPLEMENTARY PRACTICES IN HEALTH}

\section{ABSTRACT}

Integrative and Complementary Health Practices (Pics) aim to provide a holistic approach, with the aim of promoting health, and providing a good balance between technology, science and humanization. Thus, this article aims to assess the perception of future graduates of the pharmacy course on the role of the pharmacist in Pics. A field research was carried out at a Faculty of Pharmacy in Cariri Cearense. Currently, the college is approved by 10 classes of the pharmacy course. The sample used in the study was composed of students from the ninth and tenth semesters of the Pharmacy course. The results of the study show that $85.3 \%$ of academics have knowledge about Pics, however, many are not sure about their knowledge about them. It was possible to observe that students would indicate homeopathy, acupuncture, floral therapy and medicinal plants to treat certain diseases, which may be possible by pharmacists according to specific specifications. Finally, it is considered that the results of this work can contribute by adding values on the evidenced findings, assisting in the discussion of new studies on integrative and complementary practices.

Keywords: Knowledge; pharmacy students; complementary therapies.

RECEBIDO EM: 18/1/2021

MODIFICAÇÕES SOLICITADAS EM: 22/3/2021

ACEITO EM: 20/5/2021

\footnotetext{
1 Faculdade São Francisco da Paraíba. Cajazeiras/PB, Brasil.

2 Faculdade de Medicina Estácio de Juazeiro do Norte. Juazeiro do Norte/CE, Brasil.

3 Autora correspondente. Colégio e Curso Masters Gold. Avenida Rotary - Jardim Oásis. Cajazeiras/PB, Brasil. CEP 58900000. http://lattes.cnpq.br/5041426851854678. https://orcid.org/0000-0003-1568-7231. anaemiliaformiga@ hotmail.com
} 


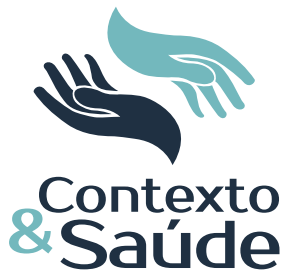

\section{INTRODUÇÃO}

As Práticas Integrativas e Complementares em Saúde (Pics) são tratamentos terapêuticos que surgem como uma forma de acréscimo para a medicina tradicional em que as mesmas são empregadas para melhorar a qualidade de vida do paciente, cooperando para o bem-estar do indivíduo e, consequentemente, impedir diversas enfermidades, como as doenças crônicas e contribuindo assim para o autocuidado, promoção e conservação da saúde. Ressalta-se que os profissionais devem ter conhecimentos técnico-científicos das práticas e assim promover um diálogo com os usuários. ${ }^{1}$

As Pics foram inseridas no Sistema Único de Saúde (SUS) a partir da Política Nacional de Práticas Integrativas Complementares (PNPIC), autorizada pela Portaria de no 971 de 3 de maio de 2006, e têm como finalidade promover a saúde, a qualidade de vida e a efetividade e uso racional e seguro de medicamentos. ${ }^{2}$

Essas práticas podem ser ofertadas na Atenção Primária à Saúde ou centros especializados em que são encontradas múltiplas opções terapêuticas e dispondo da liberdade de escolher o tratamento de acordo com sua patologia, suas culturas, crenças, simpatias e planejando um maior bem-estar. ${ }^{3}$

As práticas integrativas têm benefícios comprovados para a saúde do usuário, tais como: redução da ansiedade, estresse, enxaqueca, resfriados, diminuição de dores e tensão muscular e melhoria na qualidade do sono. ${ }^{4} \mathrm{Em}$ relação a sua aplicação, as práticas podem ser utilizadas em diferentes quadros clínicos, por exemplo em casos de usuários que fazem tratamentos para câncer, doenças crônicas (diabetes e hipertensão) e dores musculares ou de forma complementar à Medicina convencional. ${ }^{5}$

O farmacêutico é um dos profissionais da saúde qualificado para atuar nessas práticas, sendo necessário que durante a sua Graduação sejam desenvolvidas estratégias e atividades como estágios, palestras, minicursos, oficinas e projetos de extensão sobre essas Pics para que sintam-se preparados para atuar. A realização de PICs pelo farmacêutico está assegurado no Conselho Federal de Farmácia (CFF) na resolução de número 353, de 23 de agosto de 2000 (acupuntura); resolução de $n^{\circ} 601$, de 26 de novembro de 2014 (homeopatia); resolução de $n^{\circ} 477$ de 28 de maio de 2008 (plantas medicinais e fitoterápicos); resolução de $n^{\circ} 611$, de 29 de maio de 2015 (floralterapia). ${ }^{6-9}$

Pode-se destacar a assistência farmacêutica, a política nacional de práticas integrativas e complementares em saúde e o uso racional de medicamentos como políticas que trabalham de maneira preventiva, além de diminuir os custos e minimizar danos à saúde. As estratégias dessas práticas buscam instigar os mecanismos naturais de prevenção e recuperação da saúde por meio da tecnologia de forma eficaz e segura, com ênfase no atendimento humanizado, bem como a integração homem, meio ambiente e sociedade. ${ }^{6-9}$

Nesse contexto, esta pesquisa teve como objetivo avaliar a percepção de concluintes do curso de Farmácia sobre o papel do farmacêutico nas Práticas Integrativas e Complementares em Saúde. 


\section{METODOLOGIA}

Foi realizado um estudo transversal de caráter analítico-descritivo, com abordagem quantitativa. O presente estudo foi realizado em uma Faculdade de Farmácia no Cariri Cearense. Atualmente a faculdade é constituída por 10 turmas do curso de Farmácia, compondo um grupo acadêmico de 372 discentes adequadamente matriculados. A população dessa pesquisa foram todos alunos do curso de Farmácia da referida faculdade, e a amostra é composta por alunos do 9 o e 10 semestre, por estarem se aproximando do final do curso e já terem cursado disciplinas relacionadas às Pics. Como critério de inclusão o aluno deveria estar devidamente matriculado na instituição; só participaram da pesquisa os acadêmicos que estavam nos períodos específicos entre o $9^{\circ}$ e o $10^{\circ}$ semestre e que assinaram o Termo de Consentimento Livre e Esclarecimento (TCLE). Foram excluídos do estudo os alunos que não estavam regularmente matriculados na instituição e aqueles que se recusaram a responder ao questionário.

Os dados foram coletados no período de agosto a setembro de 2020, por meio de um questionário semiestruturado, constituído por questões objetivas e subjetivas com a finalidade de atender aos objetivos propostos.

Os dados foram organizados, analisados e explicados por meio de gráficos estatísticos e tabelas utilizando o software Microsoft Excel 2010, em que as categorias foram apresentadas em percentuais.

Esta pesquisa obedeceu aos aspectos éticos e recomendações da Resolução 510/2016, sendo aprovado pelo Comitê de Ética da Faculdade Estácio de Medicina de Juazeiro do Norte, CE, com parecer de aprovação número 3.020.857.

\section{RESULTADOS E DISCUSSÃO}

De acordo com os dados da amostra, dos 34 alunos que participaram da pesquisa a predominância foi do gênero feminino $(64,7 \%)$, em relação ao masculino $(35,3 \%)$, confirmando o achado ${ }^{10}$ que revela que as mulheres sempre buscam por qualificações e, consequentemente, ocupam um percentual maior de vagas nos cursos de Graduação.

Quando questionados se já tinham participado de alguma capacitação em Pics durante a Graduação, 82,4\% (28) dos entrevistados afirmaram que não e apenas $17,6 \%$ (6) realizaram alguma capacitação. Esses resultados vão ao encontro da pesquisa realizada por Goecks, Morsch e Da Silva ${ }^{11}$ com estudantes de nove cursos da área da saúde da Universidade em Santa Cruz do Sul (Unisc). A amostra foi composta por 130 discentes, dos quais 66 responderam que tinham alguma capacitação e 64 afirmaram que não possuíam nenhuma capacitação em Pics. É preciso que as faculdades proporcionem aos alunos alternativas como palestras, oficinas, minicursos, projetos de extensão, disciplinas optativas e obrigatórias e aulas práticas em que o acadêmico possa vivenciar a realidade e assim se sentir motivado e capacitado para atuar futuramente nas Pics. ${ }^{12}$

Em relação se já fez algum tratamento com algumas das práticas integrativas $55,9 \%$ (19) responderam que sim e 44,1\% (15) que não. Esse achado corrobora a pesquisa na qual $68 \%$ relataram ter utilizado alguma terapia, seja cor-

Editora Unijuí - Revista Contexto \& Saúde - ISSN 2176-7114 - v. 21, n. 44, out./dez. 2021 
relacionada ao tratamento farmacológico ou mesmo como forma de melhorar a qualidade de vida. ${ }^{11}$

No que diz respeito se os estudantes indicariam para algum familiar, $97,1 \%$ dos entrevistados responderam que sim. Em uma pesquisa com alunos da área da saúde da Universidade do Vale do Itajaí ${ }^{13}$, foi constatado que as práticas mais indicadas pelos acadêmicos foi a acupuntura, fitoterapia, ioga e meditação.

Com relação ao conhecimento das práticas integrativas e complementares em saúde, de forma geral observou-se que $85,3 \%$ dos acadêmicos possuem conhecimento a respeito dessas práticas, já 14,7\% não. Em relação se já utilizou algum tratamento com as Pics obteve-se os seguintes resultados: Homeopatia, $64,7 \%$ disseram que não e $35,3 \%$ que sim; acupuntura, 23,5\% expuseram que não e $76,5 \%$ que sim; terapia floral, $26,5 \%$ responderam que não e $73,5 \%$ que sim; plantas medicinais, 2,9\% relataram que não e $97,1 \%$ que sim.

Esses dados podem ser compatíveis com o da pesquisa em que os participantes da pesquisa, quando questionados se já utilizaram a homeopatia como terapia, revelaram que $96,4 \%$ não utilizaram e 3,6\% já usaram ${ }^{14}$. Com acupuntura $76,5 \%$ responderam que sim e 23,5\% que não (Quadro 1 ).

Quadro 1 - Distribuição da frequência segundo o conhecimento dos acadêmicos de Farmácia sobre as práticas integrativas e complementares em saúde

\begin{tabular}{|l|c|c|}
\hline & Sim & Não \\
\hline Conhecimento sobre as Pics & $85,3 \%$ & $14,7 \%$ \\
\hline \multicolumn{3}{|c|}{ Já utilizou algum tratamento? } \\
\hline Homeopático & Sim & Não \\
\hline Acupuntura & $35,3 \%$ & $64,7 \%$ \\
\hline Terapia Floral & $76,5 \%$ & $23,5 \%$ \\
\hline Plantas Medicinais Acredita na eficácia das Pics? \\
\hline \multicolumn{3}{|c|}{ Sim } \\
\hline \multicolumn{2}{|c|}{$73,5 \%$} \\
\hline Homeopático & $85,3 \%$ & Não \\
\hline Acupuntura & $91,2 \%$ & $14,7 \%$ \\
\hline Terapia Floral & $52,9 \%$ & $8,8 \%$ \\
\hline Plantas Medicinais & $97,1 \%$ & $47,1 \%$ \\
\hline
\end{tabular}

Fonte: Dados da Pesquisa, 2020.

Os dados da pesquisa são semelhantes ao estudo realizado com os acadêmicos do curso de Farmácia da Universidade Pública do Estado de Pernambuco, que constataram que os alunos possuíam algum conhecimento prévio a respeito das Pics ${ }^{15}$.

De acordo com a pesquisa de Rodrigues, Evangelista e Tescarollo ${ }^{16}$, esse conhecimento em relação às Pics podem ser justificadas pelas novas Diretrizes Curriculares Nacionais (DCNs) instituídas em 2017 para o curso de Farmácia, em 


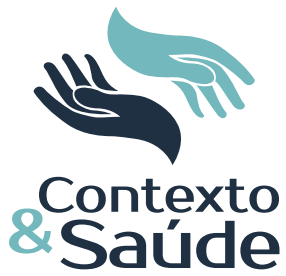

que se observa a inserção das disciplinas na grade curricular em que essas práticas integrativas podem ser ofertadas de maneira obrigatória ou optativa. Ressalta-se, no entanto, que a homeopatia sempre foi um componente curricular presente nos cursos de Graduação em Farmácia, fato que também corrobora tal conhecimento.

Esses resultados vão de encontro ao estudo realizado com os estudantes do curso de Medicina ${ }^{12}$. A amostra foi composta por 92 discentes e, quando interrogados se já haviam utilizado a prática da acupuntura, 78,25\% responderam que não e $21,73 \%$ que sim. Apesar de a maioria nunca ter usufruído dessa prática, os acadêmicos indicam o uso para os familiares. Já quanto à terapia floral, observou-se que $73,5 \%$ disseram que sim e $26,5 \%$ que não. Do Nascimento Carissimo e De Oliveira ${ }^{17}$ realizaram uma pesquisa da qual participaram 50 estudantes do Ensino Superior e destes $76 \%$ já haviam utilizado essa prática e $24 \%$ nunca fizeram o uso.

Com as plantas medicinais $97,1 \%$ afirmaram que sim e apenas 2,9\% não fizeram uso. Resultados coincidem com os encontrados na pesquisa em que $93 \%$ dos alunos entrevistados afirmaram que já utilizaram plantas medicinais para fins terapêuticos e $6,8 \%$ não terem utilizado ${ }^{18}$.

Diante dos dados analisados, observa-se que $85,3 \%$ dos discentes acreditam na eficácia do tratamento homeopático e $8,8 \%$ vê a atuação apenas de forma complementar, havendo diversas desconfianças quanto à eficácia desse tratamento. Nesse sentido, a Associação Médica Homeopática Brasileira (AMHB) em 2019 elaborou um dossiê em que apresenta "Evidências Científicas em Homeopatia" que comprovem a efetividade e as vantagens em utilizar os medicamentos, dessa forma desmistificando informações equivocadas e preconceituosas voltadas a essa prática, a qual consiste em avaliar o indivíduo dentro de uma abordagem completa. ${ }^{19}$

Questionados sobre a eficácia da acupuntura 91,2\% responderam que é eficaz e $8,8 \%$ veem apenas de forma complementar. Perfil semelhante foi encontrado no estudo em que os alunos do curso de Veterinária quando indagados sobre a eficácia observou-se que 76,9\% responderam que era sim eficaz, 21,2\% que não tinham conhecimento suficiente para opinar a respeito e 1,9\% responderam que não ${ }^{20}$.

Foi perguntado aos estudantes se acreditavam na eficácia da terapia floral: $52,9 \%$ que acredita e $29,4 \%$ que não e $17,6 \%$ apenas de forma complementar. Nesse estudo, os discentes perceberam o efeito positivo com o floral, uma vez que $32,6 \%$ que fizeram o uso dessa terapia dizem que possui sim eficácia ${ }^{17}$.

Se acreditam na eficácia das plantas medicinais $97,1 \%$ dos acadêmicos responderam que acreditam e 2,9\% que não. Um estudo realizado em uma instituição privada de Ensino Superior no Estado de Minas Gerais com alunos dos cursos de Nutrição, Farmácia, Biomedicina, Enfermagem, Psicologia e Fonoaudiologia verificou que $23 \%$ dos entrevistados responderam que as plantas medicinais são mais eficazes que medicamentos sintéticos, porém $76,6 \%$ acreditam que os medicamentos sintéticos possuem mais eficácia que as plantas ${ }^{21}$.

Editora Unijuí - Revista Contexto \& Saúde - ISSN 2176-7114 - v. 21, n. 44, out./dez. 2021 
Quando questionados sobre "Na sua concepção quais as bases das práticas integrativas e complementares"? em relação à homeopatia 67,6\% acreditam nos princípios farmacológicos não elucidados, $17,6 \%$ na energia vital das plantas e $14,7 \%$ em efeito placebo. A acupuntura com $94,1 \%$ em princípios farmacológicos não elucidados e fé e religião com 2,9\%. A terapia floral com $85,3 \%$ em princípios farmacológicos não elucidados e $14,7 \%$ na fé e religião. Já as plantas medicinais, com $82,4 \%$, estão relacionadas aos princípios farmacológicos e 17,6\% em conhecimentos empíricos.

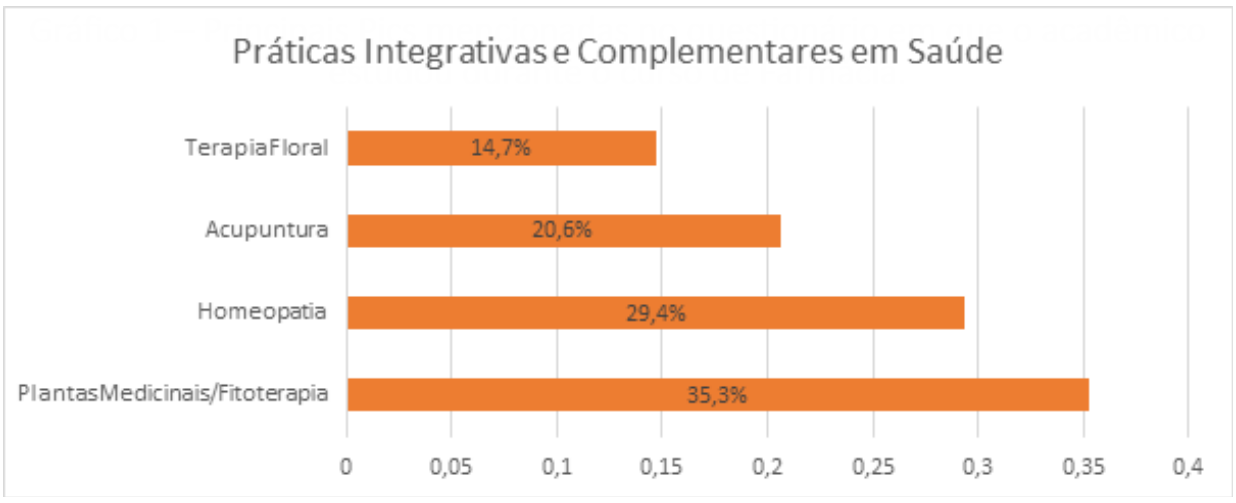

Fonte: Dados da pesquisa, 2020.

O Gráfico 1 mostra as Pics mais citadas pelos estudantes: plantas medicinais/fitoterapia com $35,3 \%$ e, em seguida a homeopatia com 29,4\%. Já em relação à acupuntura $20,6 \%$ e apenas $14,7 \%$ dos alunos citaram ter estudado a terapia floral. No estudo realizado em seis Instituições de Ensino Superior (IES) públicas do Estado do Rio de Janeiro, os autores investigaram quais as Pics ofertadas nos diferentes cursos da área da saúde e concluíram que as disciplinas ofertadas no curso de Farmácia foram a homeopatia e plantas medicinais ${ }^{22}$.

O Gráfico 2 apresenta em percentual a resposta dos participantes quanto à questão: "Como futuro profissional indicaria as Pics como terapia completar em quais doenças?" Foi observado que as PICS são reconhecidas pelos estudantes para doenças como resfriado, estresse e enxaqueca.

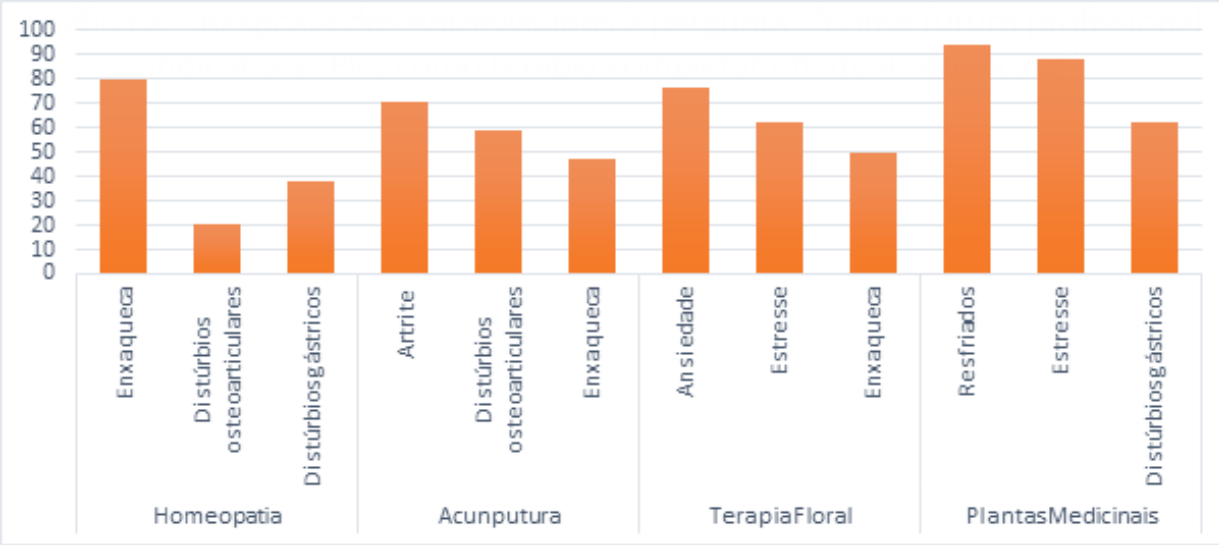


Para Campos ${ }^{23}$, as principais patologias que levaram os usuários a buscar a homeopatia foram a ansiedade $(17,4 \%)$, equilíbrio emocional $(12,3 \%)$, depressão $(5,8 \%)$, baixa autoestima e estresse (4,5\%), angústia, dor de cabeça, insônia, nervosismo, tristeza e dor na coluna (3,2\%). A acupuntura para: artrite (70,6\%); distúrbios osteoarticulares $(58,8 \%)$ e enxaqueca $(47,1 \%)$. As terapias florais foram indicadas para seguintes enfermidades: ansiedade $(76,5 \%)$, estresse $(61,8 \%)$ e enxaqueca (50\%). E, por último, em relação às plantas medicinais, $(94,1 \%)$ indicariam para resfriados, $(88,2 \%)$ enxaqueca e $(61,8 \%)$ para distúrbios gástricos.

No trabalho de Pires et al. ${ }^{24}$, realizado na comunidade de Montes Claros - MG, salienta-se que indicariam essas práticas nas respectivas doenças: resfriado (45,8\%), problemas digestivos (12,6\%), cardiovascular $(6,8 \%)$ e sistema excretor $(2,1 \%)$.

Em relação à pergunta "Como futuro profissional, você tem interesse de trabalhar com as Práticas Integrativas e Complementares em Saúde"?, Percebeu-se que os estudantes demostraram grande interesse $(67,6 \%)$ em trabalhar com as Pics e que $32,4 \%$ não têm vontade de atuar nessa área. Estudo realizado por Vieira et al. ${ }^{15}$ mostrou que $83 \%$ dos alunos desejam ter capacitação e que almejam trabalhar com elas.

Quando questionados se se sentem preparados para trabalhar com Pics após a formação, observou-se que os alunos ficaram indecisos, pois apenas $50 \%$ responderam que sim e os outros 50\% ainda não. Os estudantes apresentam uma certa insegurança para atuar com as Pics, o que supostamente estar relacionado com a falta de conhecimento específico e que as mesmas são pouco exploradas em cursos de Graduação. Sendo assim, é preciso que sejam introduzidas essas práticas nas grades curriculares como disciplinas obrigatórias.

\section{CONSIDERAÇÕES FINAIS}

De acordo com os objetivos propostos, o estudo abordou a importância das Práticas Integrativas e Complementares em Saúde voltadas aos alunos do curso de Farmácia, as quais podem proporcionar novos conhecimentos e ampliar os campos de atuação do farmacêutico, uma vez que o profissional esteja apto a realizar essas práticas, de acordo com as regulamentações exigidas pelo ConseIho Federal de Farmácia.

Foi possível observar que os alunos indicariam a homeopatia, acupuntura, terapia floral e plantas medicinais para tratar determinadas enfermidades, pois acreditam na eficácia destas práticas, porém ficaram indecisos quando questionados se se sentem preparados para trabalhar com as Pics, posto que $50 \%$ afirmaram sentir-se preparados para atuar enquanto os outros $50 \%$ ainda não estão prontos. Isto demostra que os discentes têm interesse em conhecer mais sobre estas práticas, mas a formação acadêmica ainda não prepara o aluno suficientemente para atuar nestas áreas.

Por fim, considera-se que os resultados deste trabalho possam contribuir agregando valores sobre os achados da pesquisa, auxiliando na discussão de novos 
estudos sobre as Práticas Integrativas e Complementares, uma vez que elas estão em construção e que ainda é necessário uma constante busca, com o propósito elaborar novos conhecimentos e tratamentos alternativos à Medicina convencional.

\section{REFERÊNCIAS}

${ }^{1}$ Dalmolin IS, Heidemann ITSB, Freitag VL. Prácticas integrativas y complementarias en el sistema único de salud: desvelando potencias y límites. Revista da Escola de Enfermagem da USP. [Internet]. 2019;53.

${ }^{2}$ Brasil. Ministério da Saúde (MS). Portaria N 971, de 3 de maio de 2006. Aprova a Política Nacional de Práticas Integrativas e Complementares (PNPIC) no Sistema Único de Saúde. Diário Oficial da União, Brasília, 2018. [Acesso em: 17 out. 2020]. Disponível em: http://bvsms.saude.gov.br/bvs/saudelegis/gm/2006/prt0971_03_05_2006.html

${ }^{3}$ Tesser CD, Sousa IMC, Nascimento MC. Práticas integrativas e complementares na atenção primária à saúde brasileira. Revista Saúde em debate. [Internet]. 2018;42:174-188.

${ }^{4}$ Brasil. Ministério da Saúde (MS). Práticas Integrativas e Complementares (Pics): quais são e para que servem. Brasília, 2019. [Acesso em: 17 out. 2020]. Disponível em: http://bvsms.saude.gov.br/bvs/publicacoes/politica_nacional_praticas_integrativas_ complementares_2ed.pdf

${ }^{5}$ Mendes DS, de Moraes FS, de Oliveira Lima G, da Silva PR, Cunha TA, Crossetti MDGO, Riegel F. Benefícios das práticas integrativas e complementares no cuidado de enfermagem/Benefits of integrative and complementary practices in nursing care/Beneficios de las prácticas integrativas y complementarias en el cuidado de enfermería. Journal Health NPEPS. [Internet]. 2019;4(1):302-318.

${ }^{6}$ Conselho Federal de Farmácia. Resolução N³53, de 23 de agosto de 2000. Dispõe sobre o exercício de acupuntura pelo profissional farmacêutico. Diário Oficial da União, Brasília, DF, 23 ago. de 2000. Seção 1, p. 72. [Acesso em: 25 jun. 2020]. Disponível em: https://www.cff.org.br/userfiles/file/resolucoes/353.pdf

${ }^{7}$ Conselho Federal de Farmácia. Resolução N 477, de 28 de maio de 2008. Dispõe sobre atribuições do farmacêutico no âmbito das plantas medicinais e fitoterápicos e dá outras providencias. Diário Oficial da União, Brasília, DF, 2 junho de 2008. Seção 1, p. 113-117. [Acesso em: 25 jun. 2020]. Disponível em: https://www.cff.org.br/userfiles/ file/resolucoes/res477_2008.pdf

${ }^{8}$ Conselho Federal de Farmácia. Resolução N601, de 26 novembro de 2014. Dispõe sobre as atribuições do farmacêutico no âmbito da homeopatia. Diário Oficial da União, Brasília, DF, 13 outubro de 2014. Seção 1, p. 751. [Acesso em: 25 jun. 2020]. Disponível em: https://www.cff.org.br/userfiles/file/resolucoes/601.pdf

${ }^{9}$ Conselho Federal de Farmácia. Resolução Nº 611, de 29 de maio de 2015. Dispõe sobre as atribuições clínicas do farmacêutico no âmbito da floralterapia e dá outras providencias. Diário Oficial da União, Brasília, DF, 9 de junho de 2015. [Acesso em: 25 jun. 2020]. Disponível em: https://www.legisweb.com.br/legislacao/?id=285534

10 Nóbrega JS, Silva FA, Barroso RF, Crispim DL, Oliveira CJA. Avaliação do conhecimento etnobotânico e popular sobre o uso de plantas medicinais junto a alunos de Graduação. Revista Brasileira de Gestão Ambiental. [Internet]. 2017;11(1):7-13.

${ }^{11}$ Goecks DR, Morsch LM, Da Silva CM. Formação de estudantes da área da saúde em práticas integrativas e complementares. Revista Interdisciplinar de Promoção da Saúde. [Internet]. 2019;2(2):84-91.

12 Gomes G de Souza IMS. Avaliação da concepção dos estudantes de medicina sobre as práticas médicas integrativas e complementares na Atenção Básica. Research Medical Journal, [Internet]. 2019;2(4):1-6.

${ }^{13}$ Couto AG, Binz MC, de Moraes ADJP, Caetano BDLS, da Cunha CC. Conhecimento, uso e aceitação de acadêmicos de Medicina sobre as práticas integrativas e complementares. VITTALLE - Revista de Ciências da Saúde. [Internet]. 2018;30(1):56-62. 
${ }^{14}$ Iozzi GM, da Silva RI, Homsani FH, Oliveira AP, Capella MAM, Holandino CA aceitação da terapia homeopática em hospitais da Universidade Federal do Rio de Janeiro. VITTALLE - Revista de Ciências da Saúde. [Internet]. 2018;30(1):25-35.

${ }^{15}$ Vieira LEB, da Silva IB, Matwijszyn MA, Randau K. Implantação da disciplina práticas integrativas e complementares em curso de Graduação em Farmácia. In: Congresso Nacional de Práticas Complementares em Saúde. Lagarto, SE: Editora Realize; 2017:1-6.

${ }^{16}$ Rodrigues A, Evangelista LQ, Tescarollo IL. Percepção de estudantes de Farmácia sobre aromaterapia e outras práticas integrativas complementares. Brazilian Journal of Surgery and Clinical Research. [Internet]. 2018;24(3):20-26.

${ }^{17}$ Do Nascimento Carissimo TD, De Oliveira LC. Estudo da eficácia da terapia floral em alunos submetidos a estresse. Cadernos da Escola de Saúde. [Internet]. 2012;2(8):180188.

18 Dos Santos MVJ, da Rosa CG, dos Santos PS, Rausch PC, Bellinati NVC. Práticas integrativas na promoção à saúde em doenças crônicas: uma revisão de literatura. Revista Interdisciplinar de Estudos em Saúde. [Internet]. 2019;8(2):41-56.

19 Teixeira Mz. Aos que clamam pelas evidências científicas em homeopatia. Revista de Homeopatia. [Internet]. 2017;80(1):1-3.

20 Pessoa RMA. Mapeamento do conhecimento e interesse dos estudantes do curso de Medicina Veterinária da Universidade Federal da Paraíba acerca da acupuntura areia [Trabalho de Conclusão de Curso]. Graduação em Medicina Veterinária, Universidade Federal da Paraíba; 2018.

${ }^{21}$ Damasceno EMA, Rocha RL, Pinheiro MLP, David J. O uso de plantas medicinais com atividade emagrecedora entre acadêmicos de uma instituição do norte de Minas Gerais. Revista Vozes dos Vales. [Internet]. 2015;1:1-12.

${ }^{22}$ Nascimento MCD, Romano VF, Chazan ACS, Quaresma CH. Formação em práticas integrativas e complementares em saúde: desafios para as universidades públicas. TrabaIho, Educação e Saúde. [Internet]. 2018;16(2):751-772.

${ }^{23}$ Campos FRG. Representações sociais dos usuários sobre o tratamento homeopático no município de Diamantina - MG. [Dissertação]. Mestrado Profissional em Saúde, Sociedade e Ambiente, Universidade Federal dos Vales do Jequitinhonha e Mucuri, Diamantina; 2019.

${ }^{24}$ Pires IFB, Souza AA, Feitosa MHA, Costa SM. Plantas medicinais como opção terapêutica em comunidade de Montes Claros, Minas Gerais, Brasil. Revista Brasileira de Plantas Medicinais. [Internet]. 2014;16(2):426-433. 